\title{
LOCAL DERIVATIONS ON SUBALGEBRAS OF $\tau$-MEASURABLE OPERATORS WITH RESPECT TO SEMI-FINITE VON NEUMANN ALGEBRAS
}

\author{
FARRUKH MUKHAMEDOV AND KARIMBERGEN KUDAYBERGENOV
}

\begin{abstract}
This paper is devoted to local derivations on subalgebras on the algebra $S(M, \tau)$ of all $\tau$ measurable operators affiliated with a von Neumann algebra $M$ without abelian summands and with a faithful normal semi-finite trace $\tau$. We prove that if $\mathcal{A}$ is a solid $*$-subalgebra in $S(M, \tau)$ such that $p \in \mathcal{A}$ for all projection $p \in M$ with finite trace, then every local derivation on the algebra $\mathcal{A}$ is a derivation. This result is new even in the case standard subalgebras on the algebra $B(H)$ of all bounded linear operators on a Hilbert space $H$. We also apply our main theorem to the algebra $S_{0}(M, \tau)$ of all $\tau$-compact operators affiliated with a semi-finite von Neumann algebra $M$ and with a faithful normal semi-finite trace $\tau$.
\end{abstract}

\section{INTRODUCTION}

Given an algebra $\mathcal{A}$, a linear operator $D: \mathcal{A} \rightarrow \mathcal{A}$ is called a derivation, if $D(x y)=D(x) y+x D(y)$ for all $x, y \in \mathcal{A}$ (the Leibniz rule). Each element $a \in \mathcal{A}$ implements a derivation $D_{a}$ on $\mathcal{A}$ defined as $D_{a}(x)=[a, x]=a x-x a, x \in \mathcal{A}$. Such derivations $D_{a}$ are said to be inner derivations. If the element $a$, implementing the derivation $D_{a}$, belongs to a larger algebra $\mathcal{B}$ containing $\mathcal{A}$, then $D_{a}$ is called $a$ spatial derivation on $\mathcal{A}$. A well known direction in the study of the local action of derivations is the local derivation problem. Recall that a linear map $\Delta$ of $\mathcal{A}$ is called a local derivation if for each $x \in \mathcal{A}$, there exists a derivation $D: \mathcal{A} \rightarrow \mathcal{A}$, depending on $x$, such that $\Delta(x)=D(x)$. This notion was introduced in 1990 independently by Kadison [19] and Larson and Sourour [20]. In [19] it was proved that every norm continuous local derivation from a von Neumann algebra into its dual normal bimodule is a derivation. In [20] the same result was obtained for the algebra of all bounded linear operators acting on a Banach space.

In the last decade the structure of derivations and local derivations on the algebra $L S(M)$ of all locally measurable operators affiliated with a von Neumann algebra $M$ and on its various subalgebras have been investigated by many authors (see [2, $6,6,-16,18]$ ). In [4] local derivations have been investigated on the algebra $S(M)$ of all measurable operators with respect a von Neumann algebra $M$. In particular, it was proved that, for finite type I von Neumann algebras without abelian direct summands, every local derivation on $S(M)$ is a derivation. Moreover, in the case of abelian von Neumann algebra $M$ necessary and sufficient conditions have been obtained for the algebra $S(M)$ to admit local derivations which are not derivations. In [18] local derivations have been investigated on the algebra $S(M)$ for an arbitrary von Neumann algebra $M$ and it was proved that for a von Neumann algebras without abelian direct summands every local derivation on $S(M)$ is a derivation. It should be noted that the proofs of the main result in the paper [18] are essentially based on the fact that the von Neumann algebra $M$ is a subalgebra in the considered algebras. Local and 2-local maps have been studied on different operator algebras by many authors [4, 7, 10, 11, 17,-20]. 
The present paper is devoted to local derivations on subalgebras of algebra $S(M, \tau)$ of all $\tau$-measurable operators affiliated with a von Neumann algebra $M$ with a faithful normal semi-finite trace $\tau$. Since in general case we do not assumed that these subalgebras contain the von Neumann algebra $M$, one cannot directly apply the methods of the papers [18] in this setting. Moreover, in our setting description of local derivations is an open problem. Therefore the aim of this paper to solve such a problem.

In Section 2 we give preliminaries from the theory of $\tau$-measurable operators affiliated with a von Neumann algebra $M$.

In section 3 we consider a von Neumann algebra $M$ without abelian summands and with a faithful normal semi-finite trace $\tau$. We prove that if $\mathcal{A}$ is a solid $*$-subalgebra in $S(M, \tau)$ such that $p \in \mathcal{A}$ for all projection $p \in M$ with a finite trace, then every local derivation $\Delta$ on the algebra $\mathcal{A}$ is a derivation.

In section 4 we apply the main theorem of the previous section to the Arens algebra and the algebra of all $\tau$-compact operators affiliated with a semi-finite von Neumann algebra $M$ and with a faithful normal semi-finite trace $\tau$.

\section{Algebras of $\tau$-Measurable operators}

Let $B(H)$ be the $*$-algebra of all bounded linear operators on a Hilbert space $H$, and let $\mathbf{1}$ be the identity operator on $H$. Consider a von Neumann algebra $M \subset B(H)$ with the operator norm $\|\cdot\|$ and with a faithful normal semi-finite trace $\tau$. Denote by $P(M)=\left\{p \in M: p=p^{2}=p^{*}\right\}$ the lattice of all projections in $M$ and $P_{\tau}(M)=\{p \in P(M): \tau(p)<+\infty\}$.

A linear subspace $\mathcal{D}$ in $H$ is said to be affiliated with $M$ (denoted as $\mathcal{D} \eta M)$, if $u(\mathcal{D}) \subset \mathcal{D}$ for every unitary $u$ from the commutant

$$
M^{\prime}=\{y \in B(H): x y=y x, \forall x \in M\}
$$

of the von Neumann algebra $M$.

A linear operator $x: \mathcal{D}(x) \rightarrow H$, where the domain $\mathcal{D}(x)$ of $x$ is a linear subspace of $H$, is said to be affiliated with $M$ (denoted as $x \eta M$ ) if $\mathcal{D}(x) \eta M$ and $u(x(\xi))=x(u(\xi))$ for all $\xi \in \mathcal{D}(x)$ and for every unitary $u \in M^{\prime}$.

A linear subspace $\mathcal{D}$ in $H$ is said to be strongly dense in $H$ with respect to the von Neumann algebra $M$, if

1. $\mathcal{D} \eta M$

2. there exists a sequence of projections $\left\{p_{n}\right\}_{n=1}^{\infty}$ in $P(M)$ such that $p_{n} \uparrow \mathbf{1}, p_{n}(H) \subset \mathcal{D}$ and $p_{n}^{\perp}=\mathbf{1}-p_{n}$ is finite in $M$ for all $n \in \mathbb{N}$.

A closed linear operator $x$ acting in the Hilbert space $H$ is said to be measurable with respect to the von Neumann algebra $M$, if $x \eta M$ and $\mathcal{D}(x)$ is strongly dense in $H$.

Denote by $S(M)$ the set of all linear operators on $H$, measurable with respect to the von Neumann algebra $M$. If $x \in S(M), \lambda \in \mathbb{C}$, where $\mathbb{C}$ is the field of complex numbers, then $\lambda x \in S(M)$ and the operator $x^{*}$, adjoint to $x$, is also measurable with respect to $M$ (see [23]). Moreover, if $x, y \in S(M)$, then the operators $x+y$ and $x y$ are defined on dense subspaces and admit closures that are called, correspondingly, the strong sum and the strong product of the operators $x$ and $y$, and are denoted by $x+y$ and $x * y$. It was shown in [23] that $x+y$ and $x * y$ belong to $S(M)$ and these algebraic operations make $S(M)$ a $*$-algebra with the identity 1 over the field $\mathbb{C}$. Here, $M$ is a $*$-subalgebra of $S(M)$. In what 
follows, the strong sum and the strong product of operators $x$ and $y$ will be denoted in the same way as the usual operations, by $x+y$ and $x y$.

It is clear that if the von Neumann algebra $M$ is finite then every linear operator affiliated with $M$ is measurable and, in particular, a self-adjoint operator is measurable with respect to $M$ if and only if all its spectral projections belong to $M$.

Let $\tau$ be a faithful normal semi-finite trace on $M$. We recall that a closed linear operator $x$ is said to be $\tau$-measurable with respect to the von Neumann algebra $M$, if $x \eta M$ and $\mathcal{D}(x)$ is $\tau$-dense in $H$, i.e. $\mathcal{D}(x) \eta M$ and given $\varepsilon>0$ there exists a projection $p \in M$ such that $p(H) \subset \mathcal{D}(x)$ and $\tau\left(p^{\perp}\right)<\varepsilon$. Denote by $S(M, \tau)$ the set of all $\tau$-measurable operators affiliated with $M$.

Note that if the trace $\tau$ is finite then $S(M, \tau)=S(M)$.

Consider the topology $t_{\tau}$ of convergence in measure or measure topology on $S(M, \tau)$, which is defined by the following neighborhoods of zero:

$$
V(\varepsilon, \delta)=\left\{x \in S(M, \tau): \exists e \in P(M), \tau\left(e^{\perp}\right)<\delta, x e \in M,\|x e\|<\varepsilon\right\},
$$

where $\varepsilon, \delta$ are positive numbers.

It is well-known [22] that $S(M, \tau)$ equipped with the measure topology is a complete metrizable topological $*$-algebra.

\section{LOCAL DERIVATIONS ON $*$-SUBALGEBRAS OF $\tau$-MEASURABLE OPERATORS}

Recall that $*$-subalgebra $\mathcal{A} \subset S(M, \tau)$ is said to be solid, if $x \in S(M, \tau)$ and $y \in \mathcal{A}$ satisfy $|x| \leq|y|$, then $x \in \mathcal{A}$.

The main result of this section is the following

Theorem 3.1. Let $M$ be a semi-finite von Neumann algebra without abelian direct summands and let $\tau$ be a faithful normal semi-finite trace on $M$. Suppose that $\mathcal{A}$ is a solid $*$-subalgebra in $S(M, \tau)$ such that $p \in \mathcal{A}$ for all $p \in P_{\tau}(M)$. Then every local derivation $\Delta$ on the algebra $\mathcal{A}$ is a derivation.

For the proof of this theorem we need several lemmata.

Let $M$ be a von Neumann algebra and let $x \in S(M, \tau)$. The projection

$$
r(x)=\inf \{p \in P(M): x p=x\}
$$

is called the right support of the element $x$, and the projection

$$
l(x)=\inf \{p \in P(M): p x=x\}
$$

is called its left support. The projection $s(x)=r(x) \vee l(x)$ is called the support of the element $x$. For *-subalgebra $\mathcal{A} \subset S(M, \tau)$ denote

$$
\mathcal{F}_{\tau}(\mathcal{A})=\left\{x \in \mathcal{A}: s(x) \in P_{\tau}(M)\right\} .
$$

The following properties of $\mathcal{F}_{\tau}(\mathcal{A})$ directly follow from the definition.

Lemma 3.2. Let $\mathcal{A}$ be a $*$-subalgebra in $S(M, \tau)$. Then the following assertions are equivalent:

(1) $x \in \mathcal{F}_{\tau}(\mathcal{A})$;

(2) $\exists p \in P_{\tau}(M)$ such that $p x=x$;

(3) $\exists p \in P_{\tau}(M)$ such that $x p=x$;

(4) $\exists p \in P_{\tau}(M)$ such that $p x p=x$. 
From this lemma we immediately get

Corollary 3.3. $\mathcal{F}_{\tau}(\mathcal{A})$ is an ideal in $\mathcal{A}$.

Lemma 3.4. Let $\Delta$ be a local derivation on $\mathcal{A}$. Then $\Delta\left(\mathcal{F}_{\tau}(\mathcal{A})\right) \subset \mathcal{F}_{\tau}(\mathcal{A})$.

Proof. Take any $x \in \mathcal{F}_{\tau}(\mathcal{A})$. Then due to locality of $\Delta$ one can find a derivation $D$ on $\mathcal{A}$ such that $\Delta(x)=D(x)$. It is clear that

$$
\begin{aligned}
& l(D(x) s(x)) \preceq s(x), \\
& r(x D(s(x))) \preceq s(x),
\end{aligned}
$$

where $p \preceq q$ means that $p$ is equivalent to a subprojection of the projection $q$. Since

$$
D(x)=D(x s(x))=D(x) s(x)+x D(s(x))
$$

we have

$$
\begin{aligned}
\tau(s(D(x))) & =\tau(s(D(x) s(x)+x D(s(x)))) \leq \\
& \leq \tau(s(x) \vee l(D(x) s(x)) \vee r(x D(s(x)))) \leq \\
& \leq \tau(s(x))+\tau(s(x))+\tau(s(x))=3 \tau(s(x))
\end{aligned}
$$

i.e.

$$
\tau(s(D(x))) \leq 3 \tau(s(x)) .
$$

Thus $D(x) \in \mathcal{F}_{\tau}(\mathcal{A})$, so $\Delta(x) \in \mathcal{F}_{\tau}(\mathcal{A})$.

Therefore, $\Delta$ maps $\mathcal{F}_{\tau}(\mathcal{A})$ into itself.

Let $p \in \mathcal{A}$ be a projection. One can see that the mapping

$$
D^{(p)}: x \rightarrow p D(x) p, x \in p \mathcal{A} p
$$

is a derivation on $p \mathcal{A} p$. Now let $\Delta$ be a local derivation on $\mathcal{A}$. Take an element $x \in \mathcal{A}$ and a derivation $D$ on $\mathcal{A}$ such that $\Delta(p x p)=D(p x p)$. Then

$$
p \Delta(p x p) p=p D(p x p) p=D^{(p)}(p x p) .
$$

This means that the mapping $\Delta^{(p)}$ defined similar to (3.1) is a local derivation on $p \mathcal{A} p$.

Lemma 3.5. If $\Delta$ is a local derivation on $\mathcal{A}$, then the restriction $\left.\Delta\right|_{\mathcal{F}_{\tau}(\mathcal{A})}$ is a derivation.

Proof. Let $x, y \in \mathcal{F}_{\tau}(\mathcal{A})$. Denote

$$
p=s(x) \vee s(y) \vee s(x y) \vee s(\Delta(x)) \vee s(\Delta(y)) \vee s(\Delta(x y)) .
$$

Since $\mathcal{F}_{\tau}(\mathcal{A})$ is an ideal in $\mathcal{A}$ and $\Delta$ maps $\mathcal{F}_{\tau}(\mathcal{A})$ into itself, we obtain that the projection $p \in P_{\tau}(M)$. Consider the local derivation $\Delta^{(p)}$ on $p \mathcal{A} p$. Since $p \in \mathcal{A}$ and $\mathcal{A}$ is a solid $*$-subalgebra in $S(M, \tau)$ we get $p M p \subseteq \mathcal{A}$. Furthermore, $\mathcal{A}$ has no abelian direct summands, and therefore [18, Theorem 1] implies that $\Delta^{(p)}$ is a derivation. Taking into account that $x, y \in p \mathcal{A} p$ we obtain

$$
\Delta^{(p)}(x y)=\Delta^{(p)}(x) y+x \Delta^{(p)}(y) .
$$

By construction of the projection $p$ we have

$$
\begin{aligned}
\Delta(x y)=\Delta^{(p)}(x y) & =\Delta^{(p)}(x) y+x \Delta^{(p)}(y)= \\
& =\Delta(x) y+x \Delta(y) .
\end{aligned}
$$


This means that $\Delta$ is a derivation on $\mathcal{F}_{\tau}(\mathcal{A})$. This completes the proof.

Remark 3.6. Let $y \in \mathcal{A}$ and $y p=0$ for all $p \in P_{\tau}(M)$. Since the map $x \mapsto y x y^{*}$ is positive and monotone continuous, taking $p \uparrow \mathbf{1}$ in ypy $y^{*}=0$, we obtain that y $y^{*}=0$. Therefore $y=0$.

Proof of Theorem 3.1 We shall show that

$$
\Delta(x y)=\Delta(x) y+x \Delta(y)
$$

for all $x, y \in \mathcal{A}$. We consider the following two cases.

CASE 1 . Let $x \in \mathcal{F}_{\tau}(\mathcal{A})$ and $y \in \mathcal{A}$. Since $\mathcal{F}_{\tau}(\mathcal{A})$ is an ideal in $\mathcal{A}$ and $\Delta$ maps $\mathcal{F}_{\tau}(\mathcal{A})$ into itself, we obtain that the projection

$$
p=s(x y) \vee s(\Delta(x y)) \vee s(\Delta(x) y) \vee s(x \Delta(y))
$$

has a finite trace. Taking into account the equalities

$$
x y p=x y, \Delta(x y) p=\Delta(x y)
$$

and Lemma 3.5 we obtain

$$
\begin{aligned}
\Delta(x y) & =\Delta(x y p)=\Delta(x y) p+x y \Delta(p)= \\
& =\Delta(x y)+x y \Delta(p),
\end{aligned}
$$

i.e. $x y \Delta(p)=0$.

Further

$$
\begin{aligned}
x \Delta(y p) p & =x \Delta(y p p)-x y p \Delta(p)= \\
& =x \Delta(y p)-x y \Delta(p)=x \Delta(y p),
\end{aligned}
$$

i.e.

$$
x \Delta(y p) p=x \Delta(y p) .
$$

Now taking into account (3.2), the equalities

$$
x y(\mathbf{1}-p)=0, x \Delta(y) p=x \Delta(y)
$$

and the linearity of $\Delta$ we have

$$
\begin{aligned}
x \Delta(y) & =x \Delta(y) p=x \Delta(y p+y(\mathbf{1}-p)) p= \\
& =x \Delta(y p) p+x \Delta(y(\mathbf{1}-p)) p=x \Delta(y p) p+x D(y(\mathbf{1}-p)) p= \\
& =x \Delta(y p) p+x D(y(\mathbf{1}-p) p)-x y(\mathbf{1}-p) D(p)=x \Delta(y p),
\end{aligned}
$$

where $D$ is a derivation on $\mathcal{A}$ such that $\Delta(y(\mathbf{1}-p))=D(y(\mathbf{1}-p))$. Consequently

$$
x \Delta(y p)=x \Delta(y) .
$$

Finally we obtain that

$$
\begin{aligned}
\Delta(x y) & =\Delta(x y p)=\Delta(x) y p+x \Delta(y p)= \\
& =\Delta(x) y+x \Delta(y) .
\end{aligned}
$$

Similar as above we can check the case $x \in \mathcal{A}$ and $y \in \mathcal{F}_{\tau}(\mathcal{A})$. 
CASE 2. Let $x, y \in \mathcal{A}$ be arbitrary elements. Take an arbitrary $q \in P_{\tau}(M)$. By the case 1 we have

$$
\Delta(y) q=\Delta(y q)-y \Delta(q)
$$

Taking into account this equality and the case 1 we obtain

$$
\begin{aligned}
\Delta(x y) q & =\Delta(x y q)-x y \Delta(q)= \\
& =\Delta(x) y q+x \Delta(y q)-x y \Delta(q)= \\
& =\Delta(x) y q+x[\Delta(y q)-y \Delta(q)]= \\
& =\Delta(x) y q+x \Delta(y) q,
\end{aligned}
$$

i.e.

$$
\Delta(x y) q=[\Delta(x) y+x \Delta(y)] q
$$

for all $q \in P_{\tau}(M)$. Taking into account Remark 3.6 we obtain

$$
\Delta(x y)=\Delta(x) y+x \Delta(y) .
$$

The proof is complete.

We stress that Theorem 3.1 is new even in the case of type $\mathrm{I}_{\infty}$ von Neumann factors.

For a Hilbert space $H$ by $\mathcal{F}(H)$ we denote the algebra of all finite rank operators in $B(H)$. Recall that a standard operator algebra is any subalgebra of $B(H)$ which contains $\mathcal{F}(H)$.

Theorem 3.1 implies the following result.

Corollary 3.7. Let $H$ be a Hilbert space and let $\mathcal{U}$ be a standard algebra in $B(H)$. Then any local derivation $\Delta: \mathcal{U} \rightarrow \mathcal{U}$ is a spatial derivation and implemented by an element $a \in B(H)$.

Remark 3.8. A similar result for local derivations on $B(X)$, where $X$ is a Banach space, has been obtained in [17 Corollar 3.7] under the additional assumption of continuity of the map with respect to the weak operator topology.

Remark 3.9. We note that if one replaces $S(M, \tau)$ with $S(M)$ all the results will remain true. In this case $\mathcal{F}_{\tau}(\mathcal{A})$ is replaced by the set of finite projections of $\mathcal{A}$ and instead of $\tau$ is used the dimension function.

\section{LOCAL DERIVATIONS ON ALGEBRA $\tau$-COMPACT OPERATORS AND ARENS ALGEBRAS}

In this section we shall consider a local derivations on algebras $\tau$-compact operators and on Arens algebras, respectively.

4.1. algebra of $\tau$-compact operators. In this subsection we shall consider an algebra of $\tau$-compact operators.

In the algebra $S(M, \tau)$ consider the subset $S_{0}(M, \tau)$ of all operators $x$ such that given any $\varepsilon>0$ there is a projection $p \in P(M)$ with $\tau\left(p^{\perp}\right)<\infty, x p \in M$ and $\|x p\|<\varepsilon$. The elements of $S_{0}(M, \tau)$ is called $\tau$-compact operators with respect to $M$ and $\tau$. It is known [21] that $S_{0}(M, \tau)$ is a solid $*$-subalgebra in $S(M, \tau)$ and a bimodule over $M$, i.e. $a x, x a \in S_{0}(M, \tau)$ for all $x \in S_{0}(M, \tau)$ and $a \in M$. Note that if $M=B(H)$ and $\tau=t r$, where $t r$ is the canonical trace on $B(H)$, then $S_{0}(M, \tau)=K(H)$, where $K(H)$ is the ideal of compact operators from $B(H)$.

The following properties of the algebra $S_{0}(M, \tau)$ are known (see [24]): 
Let $M$ be a von Neumann algebra with a faithful normal semi-finite trace $\tau$. Then

1) $S(M, \tau)=M+S_{0}(M, \tau)$;

2) $S_{0}(M, \tau)$ is an ideal in $S(M, \tau)$.

Note that if the trace $\tau$ is finite then

$$
S_{0}(M, \tau)=S(M, \tau)=S(M) .
$$

It is well-known [24] $S_{0}(M, \tau)$ equipped with the measure topology is a complete metrizable topological *-algebra.

It is clear that $p \in S_{0}(M, \tau)$ for all $p \in P_{\tau}(M)$.

Theorem 3.1 implies the following result.

Theorem 4.1. Let $M$ be a semi-finite von Neumann algebra without abelian direct summands and let $\tau$ be a faithful normal semi-finite trace on $M$. Then every local derivation $\Delta$ on the algebra $S_{0}(M, \tau)$ is a derivation.

Remark 4.2. If $M$ is an abelian von Neumann algebra with a faithful normal semi-finite trace $\tau$ such that the lattice $P(M)$ of projections in $M$ is not atomic, then the algebra $S_{0}(M, \tau)$ admits a local derivation which is not a derivation (see [4] Theorem 3.2]).

In [9. Theorem 4.9] it was proved that in the case when $M$ is a properly infinite von Neumann algebra with a faithful normal semi-finite trace $\tau$, then any derivation $D$ on $S_{0}(M, \tau)$ is a spatial derivation and implemented by an element $a \in S(M, \tau)$. Therefore Theorem 4.1 implies that

Theorem 4.3. If $M$ is a properly infinite von Neumann algebra with a faithful normal semi-finite trace $\tau$, then any local derivation $\Delta: S_{0}(M, \tau) \rightarrow S_{0}(M, \tau)$ is a spatial derivation and implemented by an element $a \in S(M, \tau)$.

4.2. Arens algebras. Now we are going to consider Arens algebras associated with a von Neumann algebra and a semi-finite faithful normal trace.

Let $M$ be a von Neumann algebra with a faithful normal semi-finite trace $\tau$.

Take $x \in S(M, \tau), x \geq 0$ and let $x=\int_{0}^{\infty} \lambda d e_{\lambda}$ be it spectral resolution. Denote $\tau(x)=\sup _{n \geq 1} \int_{0}^{n} \lambda d \tau\left(e_{\lambda}\right)$.

Given $p \geq 1$ put $L^{p}(M, \tau)=\left\{x \in S(M, \tau): \tau\left(|x|^{p}\right)<\infty\right\}$. It is known [21] that $L^{p}(M, \tau)$ is a Banach space with respect to the norm

$$
\|x\|_{p}=\left(\tau\left(|x|^{p}\right)\right)^{1 / p}, \quad x \in L^{p}(M, \tau) .
$$

Consider the intersection

$$
L^{\omega}(M, \tau)=\bigcap_{p \geq 1} L^{p}(M, \tau) .
$$

It is proved in [1] that $L^{\omega}(M, \tau)$ is a locally convex complete metrizable $*$-algebra with respect to the topology $t$ generated by the family of norms $\left\{\|\cdot\|_{p}\right\}_{p \geq 1}$. The algebra $L^{\omega}(M, \tau)$ is called a (non commutative) Arens algebra.

Note that $L^{\omega}(M, \tau)$ is a solid *-subalgebra in $S(M, \tau)$ and if $\tau$ is a finite trace then $M \subset L^{\omega}(M, \tau)$.

Further consider the following spaces

$$
L_{2}^{\omega}(M, \tau)=\bigcap_{p \geq 2} L^{p}(M, \tau)
$$


and

$$
M+L_{2}^{\omega}(M, \tau)=\left\{x+y: x \in M, y \in L_{2}^{\omega}(M, \tau)\right\} .
$$

It is known [2] that $L_{2}^{\omega}(M, \tau)$ and $M+L_{2}^{\omega}(M, \tau)$ are a $*$-algebras and $L^{\omega}(M, \tau)$ is an ideal in $M+$ $L_{2}^{\omega}(M, \tau)$.

Note that if $\tau(\mathbf{1})<\infty$ then $M+L_{2}^{\omega}(M, \tau)=L_{2}^{\omega}(M, \tau)=L^{\omega}(M, \tau)$.

It is known [2, Theorem 3.7] that if $M$ is a von Neumann algebra with a faithful normal semi-finite trace $\tau$ then any derivation $D$ on $L^{\omega}(M, \tau)$ is spatial, moreover it is implemented by an element of $M+L_{2}^{\omega}(M, \tau)$, i. e.

$$
D(x)=a x-x a, \quad x \in L^{\omega}(M, \tau)
$$

for some $a \in M+L_{2}^{\omega}(M, \tau)$. In particular, if $M$ is abelian, then any derivation on $L^{\omega}(M, \tau)$ is zero.

Note that $p \in L^{\omega}(M, \tau)$ for all $p \in P_{\tau}(M)$.

We need the following auxiliary result.

Lemma 4.4. Let $M$ be a semi-finite von Neumann algebra with a faithful normal semi-finite trace $\tau$ and with the center $Z(M)$. Then every local derivation $\Delta$ on the algebra $L^{\omega}(M, \tau)$ is necessarily $P(Z(M))$ homogeneous, i.e.

$$
\Delta(z x)=z \Delta(x)
$$

for any central projection $z \in P(Z(M))=P(M) \cap Z(M)$ and for all $x \in L^{\omega}(M, \tau)$.

Proof. Take $z \in P(Z(M))$ and $x \in L^{\omega}(M, \tau)$. For the element $z x$ by the definition of the local derivation $\Delta$ there exists a derivation $D_{a}$ on $L^{\omega}(M, \tau)$ of the form (4.1) such that $\Delta(z x)=D_{a}(z x)$. Since the projection $z$ is central, one has that

$$
D_{a}(z x)=[a, z x]=z[a, x]=z D_{a}(x) .
$$

Multiplying the equality $\Delta(z x)=D_{a}(z x)$ by $z$ we obtain

$$
z \Delta(z x)=z D_{a}(z x)=z D_{a}(x)=D_{a}(z x)=\Delta(z x),
$$

i.e.

$$
(1-z) \Delta(z x)=0 .
$$

Replacing $z$ by $\mathbf{1}-z$ one finds

$$
z \Delta((\mathbf{1}-z) x)=0 .
$$

Therefore by the linearity of $\Delta$ we have

$$
z \Delta(x)=z \Delta(z x)+z \Delta((\mathbf{1}-z) x)=z \Delta(z x)=\Delta(z x),
$$

and thus $z \Delta(x)=\Delta(z x)$. The proof is complete.

Theorem 4.5. Let $M$ be a semi-finite von Neumann algebra with a faithful normal semi-finite trace $\tau$. Then any local derivation $\Delta$ on the algebra $L^{\omega}(M, \tau)$ is a spatial derivation of the form (4.1).

Proof. Let $M$ be a semi-finite von Neumann algebra. There exist mutually orthogonal central projections $z_{1}, z_{2}$ in $M$ with $z_{1}+z_{2}=1$ such that

- $z_{1} M$ is abelian;

- $z_{2} M$ has no abelian summands. 
By Lemma 4.4 the operator $\Delta$ maps $z_{i} L^{\omega}(M, \tau) \equiv L^{\omega}\left(z_{i} M, \tau_{i}\right)$ into itself for $i=1,2$, where $\tau_{i}$ is the restriction of $\tau$ on $z_{i} M(i=1,2)$. As it was mentioned above $z_{1} \Delta$ is zero. By Theorem 3.1 we obtain that $\Delta=z_{2} \Delta$ is a derivation. The proof is complete.

Note that if the trace $\tau$ is finite Theorem 4.5 it was given in [11, Theorem 2.1].

\section{ACKNOWLEDGMENTS}

The second named author (K.K.) acknowledges the MOHE grant ERGS13-024-0057 for support, and International Islamic University Malaysia for kind hospitality.

\section{REFERENCES}

[1] R. Z. Abdullaev, The dual space for Arens algebra, Uzbek Math. J. 2 (1997), 3-7.

[2] S. Albeverio, Sh.A. Ayupov, K.K. Kudaybergenov, Non commutative Arens algebras and their derivations, J. Funct. Anal. 253 (2007), 287-302.

[3] S. Albeverio, Sh.A. Ayupov, K.K. Kudaybergenov, Structure of derivations on various algebras of measurable operators for type I von Neumann algebras, J. Funct. Anal. 256 (2009), 2917-2943.

[4] S. Albeverio, Sh.A. Ayupov, K.K. Kudaybergenov, B.O. Nurjanov, Local derivations on algebras of measurable operators, Comm. in Contem. Math. 13 (2011), 643-657.

[5] Sh.A. Ayupov, K.K. Kudaybergenov, Derivations on algebras of measurable operators, Infin. Dimens. Anal. Quantum Probab. Relat. Top. 13 (2010), 305-337.

[6] Sh. A. Ayupov, K. K. Kudaybergenov, Additive derivations on algebras of measurable operators, J. Operator Theory, 67 (2012) 495-510.

[7] Sh. A. Ayupov, K. K. Kudaybergenov, 2-Local derivations and automorphisms on $B(H)$, J. Math. Anal. Appl. 395 (2012), 15-18.

[8] Sh.A. Ayupov, K.K. Kudaybergenov, Innerness of continuous derivations on algebras of measurable operators affiliated with finite von Neumann algebras, J. Math. Anal. Appl., 408 (2013) 256-267.

[9] Sh.A. Ayupov, K.K. Kudaybergenov, Spatiality of derivations on the algebra of $\tau$-compact operators, Integr. Equ. Oper. Theory, 77 (2013) 581-598.

[10] Sh.A. Ayupov, K.K. Kudaybergenov, A.K. Alauadinov, 2-Local derivations on matrix algebras over commutative regular algebras, Linear Algebra and Appl., 439 (2013) 1294-1311.

[11] Sh. A. Ayupov, K. K. Kudaybergenov, B. O. Nurjanov, A. K. Alauadinov, Local and 2-local derivations on noncommutative Arens algebras, Math. Slovaca (to appear), preprint available at http://arxiv.org/abs/1110.1557.

[12] A. F. Ber, V. I. Chilin, F. A. Sukochev, Non-trivial derivation on commutative regular algebras, Extracta Math., 21 (2006) 107-147.

[13] A. F. Ber, B. de Pagter, F. A. Sukochev, Derivations in algebras of operator-valued functions, J. Operator Theory, 66 (2011), 261-300.

[14] A. F. Ber, Continuity of derivations on properly infinite $*$-algebras of $\tau$-measurable operators, Math. Notes, 90 (2011), 758-762.

[15] A. F. Ber, V. I. Chilin, F. A. Sukochev, Continuity of derivations in algebras of locally measurable operators. Integr. Equ. Oper. Theory, 75 (2013), 527-557.

[16] A. F. Ber, Continuous derivations on *-algebras of $\tau$-measurable operators are inner. Math. Notes, 93 (2013), 654-659.

[17] M. Brešar, P. Šemrl, Mapping which preserve idempotents, local automorphisms, and local derivations, Can. J. Math. 45 (1993), 483-496.

[18] D. Hadwin, J. Li, X. Li, X. Ma, Local derivations on rings containing a von Neumann algebra, arXiv:1311.0030

[19] R. V. Kadison, Local derivations, J. Algebra. 130 (1990), 494-509.

[20] D. R. Larson, A. R. Sourour, Local derivations and local automorphisms of $B(X)$, Proc. Sympos. Pure Math. 51. Providence, Rhode Island, 1990, Part 2, 187-194. 
[21] M. A. Muratov, V. I. Chilin, Algebras of measurable and locally measurable operators, Institute of Mathematics Ukrainian Academy of Sciences 2007.

[22] E. Nelson, Notes on non-commutative integration, J. Funct. Anal. 15 (1974) 103-116.

[23] I. A. Segal, A non-commutative extension of abstract integration, Ann. of Math. 57 (1953), 401-457.

[24] A. Stroh, G. P. West, $\tau$-compact operators affiliated to a semifinite von Neumann algebra, Proc. Royal Irish Acad. 93 (1993), 73-86.

(Farrukh Mukhamedov) Department of Computational \& Theoretical Sciences, Faculty of Science, International Islamic University Malaysia, P.O. Box, 141, 25710, KuANTAN, PAHANG, Malaysia

E-mail address: far75m@yandex.ru farrukh_meiium.edu.my

(Karimbergen Kudaybergenov) DePartment of Mathematics, KaraKalPaK STATE UniVERSity, 230113 NukUS, UZBEKISTAN.

E-mail address: karim2006@mail.ru 